\title{
Android Based Remote Surveillance System and Content Sharing Between PC And Mobile
}

\author{
S. A. Ahirrao \\ Sandip Foundation (SIEM) \\ Nashik, India
}

\author{
Sayali S. Ballal \\ Sandip Foundation (SIEM) \\ Nashik, India
}

\author{
Divya K. Sawant \\ Sandip Foundation (SIEM) \\ Nashik, India
}

\author{
Harsha H. Chahira \\ Sandip Foundation (SIEM) \\ Nashik, India
}

\author{
Amisha A. Savaliya \\ Sandip Foundation (SIEM) \\ Nashik, India
}

\begin{abstract}
This paper presents the Video Surveillance System architecture to improve surveillance applications which are best on the use of the service oriented paradigm, with Android Smartphone's as user terminal. It increases the flexibility of the system. This system allows to access the videos from different localization anywhere and anytime.

This paper also presents the content sharing, which is based on peer to peer technology [3]. In this, mobile will be considered as one peer of the network \& the PC will be considered as the other peer of the network [4]. Using internet connection at both the ends, we can connect two discrete system in a network \& peer to peer networking is possible. This system is used for applications like Uploading or downloading the files which is there in a remote computer, Storing the files and images in a remote computer through the mobile phone and also we can control remote computer by using mobile phone.
\end{abstract}

Keywords: Android; Surveillance; Peer to Peer; Internet Connection.

\section{INTRODUCTION}

In our day-to-day life, people don't want to waste their valuable time in monitoring the videos of institutes or any organization for security purpose. People want everything to happen at their door step without making so much effort. In this system the user can view/share the videos, also we can upload/download the files from anywhere in the world. That means we want multitasking facilities to happen at any remote area[1]

In video surveillance system, videos can be monitored from anywhere through mobile phone with web camera which can be inbuilt web camera or external using android. There are different surveillance methodologies like alarm system, CCTV, PC based video system are use to ensure the security[1]. But using all these systems, it is not possible for a user to monitor the security of his or her institute (Location) when they are outside. Means using all these systems, the person requires to continuously monitoring the video for security purpose and this is main drawback. Because now-a-days anybody can communicate with anyone at any time around the globe with the help of mobile phone technology. By using mobile, people can monitor the videos of their institution/organization even they are at outside. This video is recorded in the system by using web camera \& people can monitor the videos through the mobile. So this will ensure more security.
The security problem is resolved by video surveillance system, but there exits also another problem which we are overcoming like insufficient storage in mobile phone. This problem is resolved by content sharing system. Sometimes whenever there is less memory space available to store the data in to our mobile phones, at that time we can transfer the data to remote PC in the network and free some memory from our mobile phone by uploading some of the files in remote PC which is at remote location. This helps us to quickly access the data instead of manually connecting our mobile phone to some PC and transfer data. Also by using content sharing system, we can transfer the data from Mobile to PC and from PC to Mobile. We can view the information through the mobile from remote $\mathrm{PC}$ from anywhere in the world[5].

Also we are providing security for Simple Users and Administrator. Simple users can access only those drives which are allocated to them during their Registration process. One simple user can not access the other simple users information as the users individually are allocated the access rights and the administrator have full access to the system.

Utilization of memory space is more when application is kept in cloud. Here instead of IP camera we are using Web camera as IP camera uses concept of cloud and cloud incurs more cost when android server is stored in cloud. Also using cloud is not affordable to many users. 
The mobile will specify the file name and the system will search the directories for the particular file name, from which the contents are viewed through the mobile. The main requirement is that the system must be switched $\mathrm{ON}$ with internet connection enabled and the mobile should have GPRS connection.[1]

\section{LITERATURE SURVEY}

In our day to day life, people want multitasking facilities to happen at their door steps. So continuously monitoring the system for security is not possible every time for them which will take more time and efforts in monitoring. We are overcoming this drawback by using Video Surveillance system by using mobile phone. Because now-adays anybody can communicate with anyone at anytime around the globe with the help of mobile phone technology. By using mobile phone, people can monitor the videos of their institute or organization when they are outside of their organization or institutes. The video is recorded in the system by using web camera and user can monitor through the mobile phone anytime. This will ensure more security. [1]

The security problem is resolved by video surveillance system, but their exists another problem which we overcoming like insufficient storage in mobile phone. This problem is resolved by content sharing system. Sometimes whenever there is less memory space into our mobile phone available to store the data, at that time we can transfer the data to remote PC in the network and free some memory from mobile $\mathrm{p}$ hone by uploading some of the files in remote PC which is at remote location. This helps us to quickly access the data, instead of manually connecting our mobile phone to some PC and transfer data. By using content sharing system, we can transfer the data from mobile to $\mathrm{PC}$ as well as from PC to mobile. We can view the information through mobile phone from remote PC from anywhere in the world.

Also we are overcoming the drawbacks of team viewer software by providing the security for simple users and administrator. Simple users can access only those drives which are allocated to them during their registration process. One simple user cannot access other simple user information as the users individually are allocated the access rights and administrator has full access to the system.

\section{THE BUILDING BLOCKS: OVERVIEW}

\subsection{System Architecture}

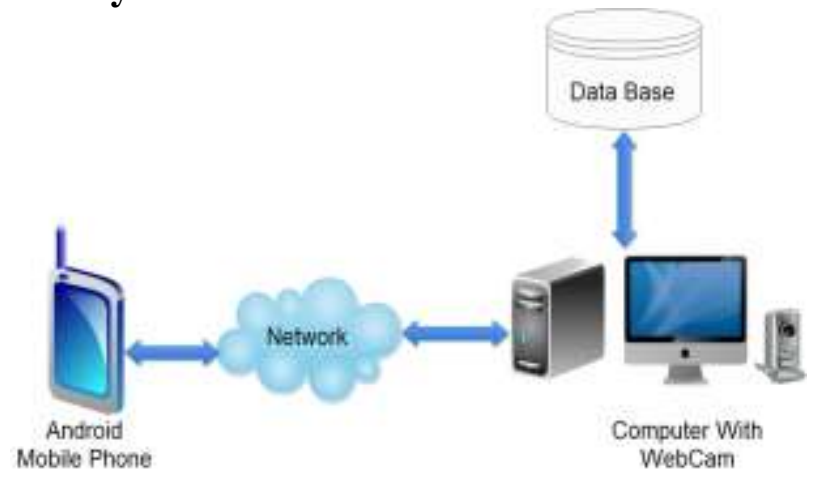

Fig 3.1: System Architecture Diagram.

As shown in Fig 3.1, Mobile and System are considered as two peers of the network. Android mobile phone is considered as a client and system with web camera is considered as a server, also internet connection is required at both the ends that is client side and server side. Database is connected to server system. Suppose user wants to access particular file, then he/she can login form his/her mobile by login id and password and request for particular file. The server computer search the particular requested file. If that file is found in database then server PC fetch that file from database and send to the user.

\subsection{Proposed System 3.2.1 Content Sharing}

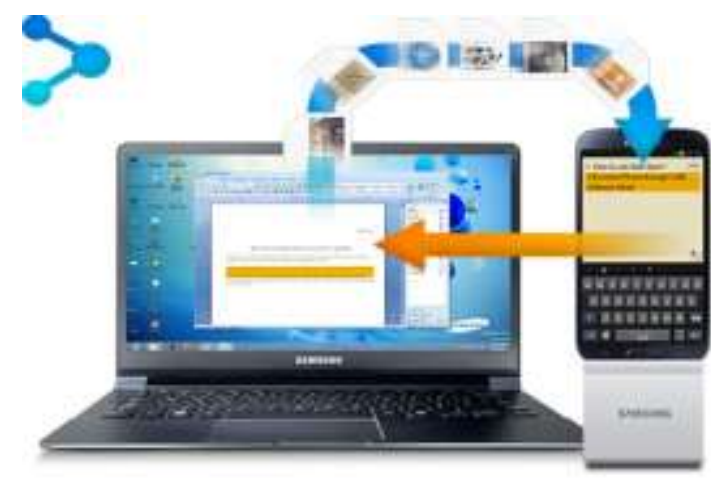

Fig 3.2.1 (i): Content Sharing.

It is a peer to peer technology. The data is transfer between PC and Mobile in a secured way. User can upload file as well as download the file which are stored in to the system. 


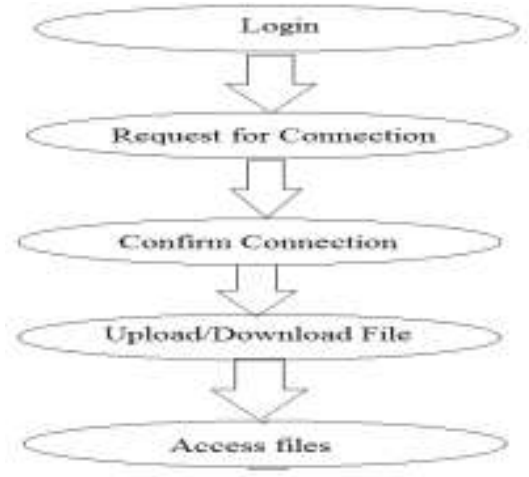

Fig 3.2.1 (ii): Algorithm for Content Sharing.

\subsubsection{Video Surveillance}

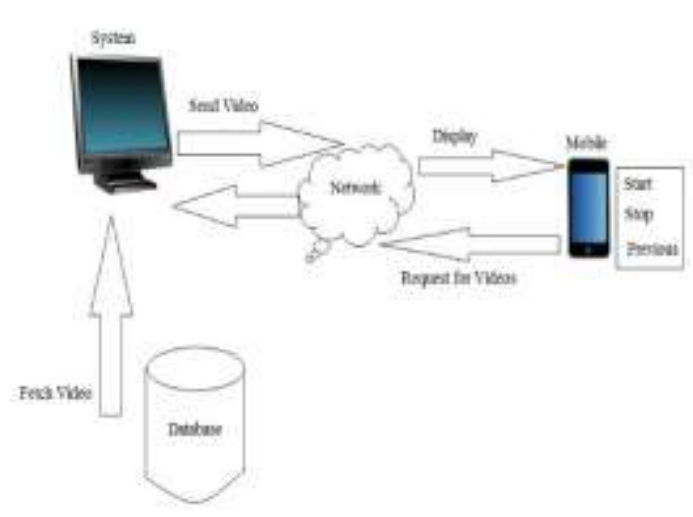

Fig 3.2.2 (i): Video Surveillance

The PC at remote location will fetch the videos from database and send those videos over the network which are displayed on mobile phone which is end user.

The process of sending and receiving video is done using socket programming and TCP protocol at the both the ends that is at client and server side.

At server side PC that is Remote PC one background thread is continuously running and this background thread slice the video which is continuously captured by the web camera.

This slice videos are being numbered and store into the database. And while fetching videos from mobile that videos are being stored into the SD card. The database will store the videos according to their date and time.

The SD card will store only the latest 10 12 videos and other videos if user wishes to see so the user can request it from the database.

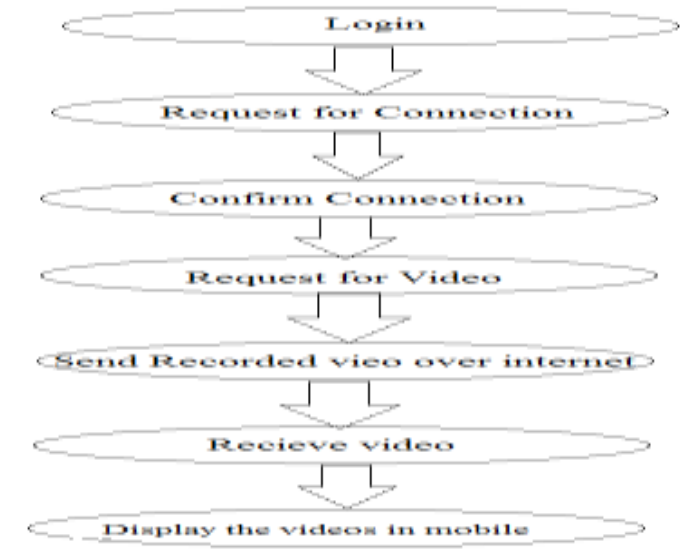

Fig 3.2.2 (ii): Algorithm for Video Surveillance.

\subsection{Overview Of Android}

The mobile application would be develop in Android. Android is a mobile Operating System which is based on Linux Kernel that is developed by Google. In android, user interface is user friendly. Android is designed mainly for touch screen mobiles such as smart phones and tablets. Android is not only designed for touch screen input, but also it has been used in games, digital cameras, electronics, etc.

The main goal of the Android project is to create a successful real-world product that improves the mobile experience for end users.

Android is a software platform developed by Google. It allows developers to write and manage code in a Java language, that uses Java libraries which are developed by Google. Java is platform-independent language as it can run on any operating system. Also java is portable and robust in nature. In this paper we are using mobile application in android and desktop application in Java.[2]

\section{MATHEMATICAL MODEL} Input:

$$
\mathrm{I}=\{\mathrm{I} 1, \mathrm{I} 2\}
$$

Where,

$$
\begin{aligned}
& \text { I1 = Video Surveillance } \\
& \text { I2= Content Sharing }
\end{aligned}
$$

I1 contains the set of input values which perform various operations such as play, pause, previous.

Operations are,

opA $=\{$ opA1, opA2, opA 3$\}$

Where, opA1= Play

$$
\begin{aligned}
& \text { opA2 }=\text { Pause } \\
& \text { opA3 }=\text { Previous }
\end{aligned}
$$


The following Venn diagram shows the relation is one to many as shown in fig 4.1

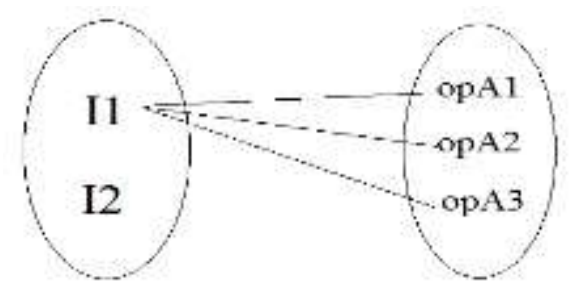

Fig 4.1

Input $\mathrm{I} 2$ contains the set of input values which performs various operations such as upload and download files.

Operations are,

$\mathrm{opB}=\{\mathrm{opB} 1, \mathrm{opB} 2\}$

Where,

$$
\begin{aligned}
& \text { opB1 }=\text { Upload } \\
& \text { opB2 }=\text { Download }
\end{aligned}
$$

The following Venn diagram shows the relation is one to many as shown in fig 4.2

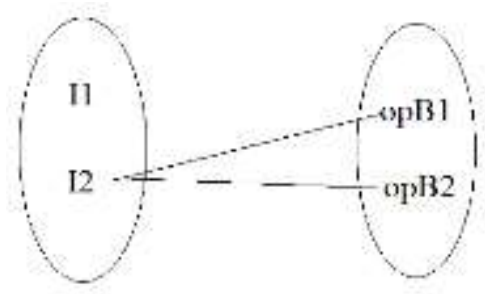

Fig 4.2

Output:

The output functions defines the output ' $\mathrm{O}$ ' of the system i.e. $\mathrm{O} 1, \mathrm{O} 2, \mathrm{O} 3$.

$$
\mathrm{O}=\{\mathrm{O} 1, \mathrm{O} 2, \mathrm{O} 3\}
$$

Where,

$$
\text { O1= Play Video }
$$

$\mathrm{O} 2=$ Uploaded File on server side PC / Remote PC

O3= Download File on client side / Mobile Phone

From the above equations (1), (2) \& (3) the following fig 4.3 depicts the set of input values operations or functions performed on it and the set of output generated based on the given Inputs.

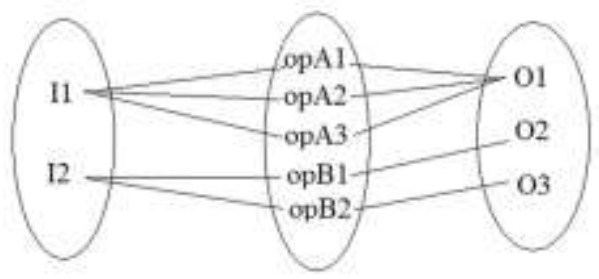

Fig 4.3

\section{ACKNOWLEDGMENTS}

We are extremely grateful to our guide Prof. S. A. Ahirrao, Assistant Professor, Department of Computer Engineering, SIEM for providing all the required resources for the successful completion of our Paper.

\section{REFERENCES}

[1] D. Shiny Irene PG Scholar," Video Surveillance System And Content Sharing Between Mobile And PC Using Android",Dept of Computer Science and Engineering RMK Engineering College, Anna University of Technology, Kavaraipettai, Chennai, Pages(s),Year 2012.

[2] Jae Kyu Lee, Jong Yeol Lee,"Android Programming Techniques for Improving Performance",IEEE 3rd International Conference on Awareness Science and Technology(iCAST),Page(s) 386-389, Year 2011.

[3] Choon Hoong Ding, Sarana Nutanong, and Rajkumar Buyya," Peer-to-Peer Networks for Content Sharing", Grid Computing and Distributed Systems Laboratory, Department of Computer Science and Software Engineering, The University of Melbourne, Australia.

[4] Jari Porras, Petri Hiirsalmi and Ari Valtaoja,"Peer-topeer Communication Approach for a Mobile Environment",Lappeenranta University of Technology P.O. Box 2053851 Lappeenranta Finland.

[5] Masahiro Hamasaki, Hideaki Takeda,"Proposal of Decentralized Information Sharing System using Local Matchmaking",Graduate Universities of Advanced Studies National Institute of Informatics 2-1-2 Hitotsubashi, Chiyoda-ku, Tokyo JAPAN. 